PHYSICAL REVIEW D 95, 109901(E) (2017)

\title{
Erratum: Detector configuration of DECIGO/BBO and identification of cosmological neutron-star binaries [Phys. Rev. D 83, 044011 (2011)]
}

\author{
Kent Yagi and Naoki Seto \\ (Received 17 April 2017; published 5 May 2017)
}

DOI: 10.1103/PhysRevD.95.109901

The shot noise for the noise spectrum of DECIGO [the first term in Eq. (5) of the original work] needs to be corrected to $6.53 \times 10^{-48}\left[1+\left(f / f_{p}\right)^{2}\right]$. From a single triangular detector unit, one can construct two L-shaped interferometers of which the noises are uncorrelated (see Fig. 2 of the original work). However, strictly speaking, the corrected noise spectrum mentioned above does not correspond to such an L-shaped interferometer. Instead, the noise spectrum for this L-shaped DECIGO interferometer is given by

$$
S_{h}^{\mathrm{DECIGO}}(f)=7.05 \times 10^{-48}\left[1+\left(\frac{f}{f_{p}}\right)^{2}\right]+4.8 \times 10^{-51}\left(\frac{f}{1 \mathrm{~Hz}}\right)^{-4} \frac{1}{1+\left(\frac{f}{f_{p}}\right)^{2}}+5.33 \times 10^{-52}\left(\frac{f}{1 \mathrm{~Hz}}\right)^{-4} \mathrm{~Hz} z^{-1} .
$$

This corresponds to rescaling the (corrected) noise spectrum in Eq. (5) of the original work by a factor $\sim 1.08$ or that in Ref. [1] by a factor $(\sqrt{3} / 2)^{-2}$. The latter is due to the fact that the effect of the angle between detector arms being $60^{\circ}$ has been accounted for in the waveform amplitude in Ref. [1] while we include such an effect in the detector noise in the original work. Figure 1 corresponds to Fig. 3 in the original work with the DECIGO noise spectrum replaced with Eq. (1) above.

The third term in Eq. (6) in the original work for the $\mathrm{BBO}$ noise spectrum needs to be corrected to $1.26 \times 10^{-51}(\mathrm{f} / 1 \mathrm{~Hz})^{-4}$.

All the calculations were carried out using the correct noise spectrum. Even if one wishes to use the above DECIGO noise spectrum, the results presented in the original work are unaffected as they are discussed in terms of the normalized signal-to-noise ratio, where the overall noise spectrum difference is irrelevant.

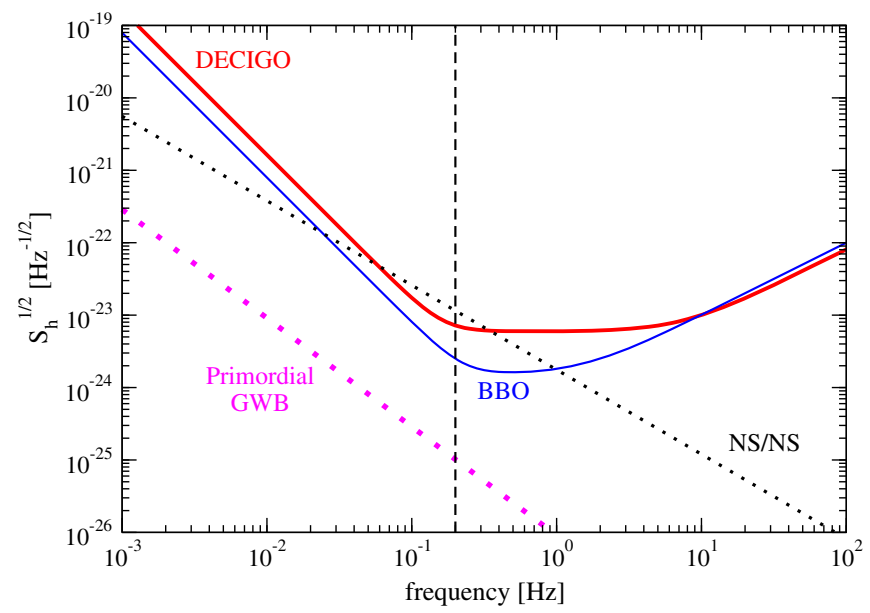

FIG. 1. The solid curves show the noise spectra for DECIGO (thick red) and Big Bang Observatory (BBO) (thin blue). Note that these noise curves are the sky-averaged ones (a factor of $\sqrt{5}$ larger than the original ones). The (black) thin dotted line represents the expected total amplitude of neutron star/neutron star (NS/NS) foreground, and the (purple) thick dotted line represents the primordial gravitational wave $(\mathrm{GW})$ background corresponding to $\Omega_{\mathrm{GW}}=10^{-16}$. The (black) dashed line at $f=0.2 \mathrm{~Hz}$ indicates the upper frequency cutoff of white dwarf/white dwarf (WD/WD) confusion noises.

[1] K. Yagi and T. Tanaka, Prog. Theor. Phys. 123, 1069 (2010). 